Received: 2 February 2017

Accepted: 21 June 2017

Published online: 31 July 2017
ENTIFIC REP

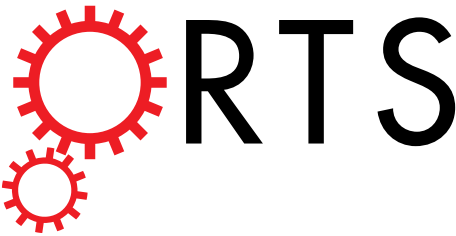

\section{OPEN An atypical strictosidine synthase, OsSTRL2, plays key roles in anther development and pollen wall formation in rice}

Ting Zou ${ }^{1,2}$, Shuangcheng $\mathrm{Li}^{1,2,3}$, Mingxing $\mathrm{Liu}^{1,2}$, Tao Wang ${ }^{1,2}$, Qiao Xiao ${ }^{1,2}$, Dan $\mathrm{Chen}^{1,2}$, Qiao $\mathrm{Li}^{1,2}$, Yanling Liang ${ }^{1,2}$, Jun Zhu ${ }^{1,2,3}$, Yueyang Liang ${ }^{1,2}$, Oiming Deng ${ }^{1,2,3}$, Shiquan Wang ${ }^{1,2,3}$, Aiping Zheng ${ }^{1,2}$, Lingxia Wang ${ }^{1,2,3}$ \& Ping $\mathrm{Li}^{1,2,3}$

Strictosidine synthase (STR) plays an important role in the biosynthesis of terpenoid indole alkaloids (TIAs) and is expressed in a range of active meristematic tissues of higher plants. STR proteins are involved in different physiological and biochemical pathways. However, the function of STR proteins in rice development remains poorly understood. In this study, we identified 21 possible STR-like (OSSTRL) family members in rice genome and found that only one gene, OsSTRL2, exhibited a pre-emergency specific florescence expression pattern. Tissue-specific expression profile analysis, $\beta$-glucuronidase histochemical (GUS) staining and RNA in situ hybridization confirmed that OsSTRL2 was highly expressed in tapetal cells and microspores. Comparative protein sequence analysis indicated that OsSTRL2 lacked the key catalytic residue found in a typical STR (STR1), although it possessed conserved $\beta$-propellers and $\alpha$-helices formed the basic structure of STR1. OsSTRL2 knockout mutant resulted to male sterility because of the defects in anther development and pollen wall formation. Subcellular localization of OsSTRL2-YFP revealed that the OsSTRL2 protein was primarily localized in the endoplasmic reticulum (ER). Therefore, OsSTRL2 is an atypical strictosidine synthase that plays crucial roles in regulating anther development and pollen wall formation in rice.

Terpenoid indole alkaloids (TIAs) are among the most important secondary metabolites in plants that play important roles in the growth and reproductive development of plants ${ }^{1}$. Over 100 different TIAs were discovered in Catharanthus roseus (periwinkle) ${ }^{2}$. Strictosidine, identified 40 years ago as the key biosynthetic precursor of TIA, is presented in a wide variety of higher plants ${ }^{1,3}$. This important molecule is generated by strictosidine synthase (STR) from tryptamine and secologanin. STR1 (GenBank accession no. P68175) is the first STR gene isolated from Rauvolfia Serpentine. STR1 catalyzes the Pictet-Spengler reaction between tryptamine and secologanin and is a key enzyme for the biosynthesis of alkaloids ${ }^{4-6}$. Recent structural characterizations reveal that STR1 is a six-bladed four-stranded $\beta$-propeller fold from the plant kingdom, and Glu-309 is the key catalytic residue in the catalysis of STR $1^{4,7}$. Fifteen and six members of the STR-like family were identified in Arabidopsis and periwinkle, respectively ${ }^{8,9}$. Among these 15 STR-like genes in Arabidopsis, four members exhibit relatively independent regulating patterns, play specific roles in the plant defense mechanism, and show higher similarity with those in Drosophila melanogaster and Caenorhabditis elegans than the other members ${ }^{10}$. STR-like members can be divided into several categories based on their different protein sequence characteristics. STR-like genes might have a variety of functions and are involved in several biochemical processes in vivo ${ }^{8,11}$. Hence, the unknown function of STR protein and its biochemical pathway should be determined by studying the isolation and expression of STR-like family members in different species.

Oryza sativa (rice) is one of the most important food crops worldwide and is an ideal model plant for monocot species ${ }^{12}$. Rice is also an excellent species for functional genomics in research because of the relatively small

${ }^{1}$ State Key Laboratory of Hybrid Rice, Sichuan Agricultural University, Chengdu, 611130, China. ${ }^{2}$ Rice Research Institute, Sichuan Agricultural University, Chengdu, 611130, China. ${ }^{3}$ Key Laboratory of Crop Genetic Resources and Improvement, Sichuan Agricultural University, Ministry of Education, Ya'an, 625014, China. Ting Zou and Shuangcheng Li contributed equally to this work. Correspondence and requests for materials should be addressed to S.L. (email: lisc926105@163.com) or P.L. (email: liping6575@163.com) 
genome size of only $400 \mathrm{Mb}^{13}$. Male fertility is crucial for rice, because it directly affects the rice yield. Male reproductive development is one of the most complex biological processes in higher plants that requires the participation of a large number of different genes. Male reproductive development can generally be divided into two stages. The first stage is the development of anther, which includes microspore mother cell (MMC) formation and microspore undergoing meiosis to form tetrads of microspores. The second stage is the formation of pollen, which includes the release of tetrads, microspore mitosis, anther tissue degeneration, anther dehiscence, and the release of mature pollen grains ${ }^{14}$. A large number of genes expressed in pre-emergency inflorescence or young flower throughout the anther development process. More than 3,000 of these genes are specifically expressed in anther before pollen maturity, whereas several of these are critical for anther and pollen development ${ }^{15}$. Any loss-function of these critical genes will lead to male sterility ${ }^{16}$.

With the completion of rice genome sequencing, a number of anther-specific genes were cloned in rice and mutated to obtain sterile male genes, such as UDT1, TDR, EAT1, TIP2, PTC1, CYP703A3, CYP704B2, DPW, and $O s A B C G 15^{17-27}$. UDT1 is expressed in early meiosis and plays an important role in the development of tapetum $^{18}$. Three basic helix-loop-helix family genes, TDR, EAT1, and TIP2, play central roles in controlling tapetum programmed cell death (PCD). Loss-function of each genes delays the degradation of the tapetum and results in pollen abortion. TIP2 is expressed at the upstream of TDR and EAT1, and can interact with TDR to form a heterodimer, and thus activate EAT1 transcription by binding to its promoter ${ }^{17,19,26,27}$. PTC1 encodes a PHD-finger protein, which is specifically expressed in tapetal cells and microspores during anther development, and controls the programmed tapetal development and functional pollen formation ${ }^{20}$. Two cytochromes, CYP703A3 and CYP704B2, play a similar role in the formation of both cuticle and exine during plant male reproductive development $^{21,22}$. $D P W$, a novel fatty acid reductase, is important for primary fatty alcohol synthesis for anther cuticle and pollen sporopollenin biosynthesis ${ }^{23,24}$. OsABCG15 functions as the ATP-binding cassette (ABC) transporter of lipid precursors in the formation of anther cuticle and pollen exine, and it is preferentially expressed in the tapetum of young anther ${ }^{25}$.

A genome-wide analysis of the rice STR-like family has not yet been reported, although the sequencing of the rice genome was completed in 10 years ago ${ }^{13}$. In the present study, we performed the first genome-wide analysis of the rice STR-like family (OsSTRL) and identified 21 possible family members in the rice genome. We also present evidence that OsSTRL2 plays important roles in anther development and pollen wall formation. Among the 21 OsSTRL members, only OsSTRL 2 showed a specific and strong transcript signal for pre-emergency inflorescence, young panicles, and anthers. Although OsSTRL 2 contains the conserved six repetitive $\beta$-propellers and three $\alpha$-helices that formed the basic structure of STR1, this protein lacks the key catalytic residue found in STR1. The knockout of OsSTRL2 caused male sterility due to the defects of the anther wall and pollen exine development. GUS-reporter gene driven by its native promoter and RNA in situ hybridization indicated that OsSTRL2 was specifically expressed in tapetal cells and microspores. Moreover, OsSTRL2 was predominantly targeted to the ER. Overall, our results suggest that OsSTRL2 is an atypical strictosidine synthase that is essential for anther development and pollen wall formation in rice; however, this protein might not act similar to a typical strictosidine synthase in its biochemical pathways.

\section{Results}

Genome-wide identification of 21 OsSTRL genes in rice. BLASTP analysis was performed with STR1 protein sequence to identify the OsSTRL members in rice. Twenty-one putative OsSTRL protein sequences were obtained (Table 1) from the MSU Rice Genome Annotation Project Database (RGAP; http://rice.plantbiology. $\mathrm{msu}$.edu/ $)^{28}$. To further confirm the reliability of these candidates, we performed conserved domain analysis by using the Simple Modular Architecture Research Tool (SMART) ${ }^{29}$. All OsSTRLs were detected within the "Str_synth" domain (PF03088.9) in their protein sequences. Through genomic distribution analysis, we found that the 21 OsSTRL members were distributed across chromosomes 1, 3, 6, 7, 8, 9, 10, 11, and 12. These genes were not found in the other three chromosomes (Table 1).

To understand the relationship among the OsSTRL genes, we used corresponding protein sequences from these genes and STR1 to perform peptide alignment by employing Clustal W (www.ebi.ac.uk/Clustalw). The result showed significant conservation within the strictosidine synthase domain among the OsSTRL proteins (Supplementary Figure 1). A neighbor-joining (NJ) phylogenetic tree was constructed by using the above multiple sequence alignment results with bootstrap analysis (1,000 replicates) (Supplementary Figure 2) to obtain clues about the evolutionary history of the OSSTRL genes. To analyze the conserved motifs of OsSTRL proteins, we employed the MEME motif search tool to investigate the shared motifs ${ }^{30}$. The OsSTRL genes were manually divided into two major groups based on the bootstrapping values of phylogram (Supplementary Figure 2) and the conserved motif distribution of OsSTRL proteins (Supplementary Figure 2). Group I is composed of four clades and three singletons, namely, OsSTRL19, OsSTRL3, and OsSTRL18 (Table 1 and Supplementary Figure 2); STR1 acted as a singleton in this group (Table 1 and Supplementary Figure 2), which is probably due to the divergence of ancient evolution between rice and Rauvolfia serpentine. By using the MEME motif search tool, we identified that: (1) motif 3 was conserved in all the members of groups I and II; and (2) motifs 2 and 3 comprised the strictosidine synthase domain in STR1 and most of OsSTRL proteins (Supplementary Figure 2).

OsSTRL2 is an atypical strictosidine synthase that is specifically expressed in the developing anther in rice. Given that protein functions are often correlated with the gene expression patterns, we performed digital expression analysis of OsSTRL members by using the RNAseq data from the Next Generation Sequencing Transcriptome Data in the RGAP (NGSTD, (http://rice.plantbiology.msu.edu/expression.shtml). OsSTRL transcripts obtained from NGSTD (Fig. 1A) suggested that two members (OsSTRL3 and OsSTRL12) had ubiquitously strong expressions, whereas the genes in clades I and II (Table 1 and Supplementary Figure 2) had relatively low expressions in the studied tissues. We manually excluded OsSTRL4 from the heat map in Fig. 1A 


\begin{tabular}{|l|l|l|l|l|l|}
\hline $\begin{array}{l}\text { Serial } \\
\text { no. }\end{array}$ & $\begin{array}{l}\text { Generic } \\
\text { name }\end{array}$ & Gene ID in RGAP & $\begin{array}{l}\text { RGAP CDS } \\
\text { Coordinates }\left(5^{\prime} \text { to 3') }\right.\end{array}$ & $\begin{array}{l}\text { Chromosamal } \\
\text { location }\end{array}$ & $\begin{array}{l}\text { Proposed clade or } \\
\text { singleton/group }\end{array}$ \\
\hline 1 & OsSTRL1 & LOC_Os01g50330 & $28892212-28891193$ & 1 & Clade II/group I \\
\hline 2 & OsSTRL2 & LOC_Os03g15710 & $8666146-8664064$ & 3 & Clade III/group I \\
\hline 3 & OsSTRL3 & LOC_Os03g53950 & $30929823-30927223$ & 3 & Singleton/group I \\
\hline 4 & OsSTRL4 & LOC_Os06g35950 & $20983248-20982674$ & 6 & Group II \\
\hline 5 & OsSTRL5 & LOC_Os06g41820 & $25081930-25080893$ & 6 & Clade I/group I \\
\hline 6 & OsSTRL6 & LOC_Os06g41830 & $25085537-25086526$ & 6 & Clade I/group I \\
\hline 7 & OsSTRL7 & LOC_Os06g41850 & $25093242-25094294$ & 6 & Clade I/group I \\
\hline 8 & OsSTRL8 & LOC_Os07g35970 & $21519953-21518519$ & 7 & Clade I/group I \\
\hline 9 & OsSTRL9 & LOC_Os07g35990 & $21532128-21531376$ & 7 & Clade I/group I \\
\hline 10 & OsSTRL10 & LOC_Os07g36040 & $21558783-21559529$ & 7 & Clade I/group I \\
\hline 11 & OsSTRL11 & LOC_Os07g36060 & $21564056-21565111$ & 7 & Clade I/group I \\
\hline 12 & OsSTRL12 & LOC_Os07g42250 & $25287511-25290131$ & 7 & Clade III/group I \\
\hline 13 & OsSTRL13 & LOC_Os08g07810 & $4371022-4372930$ & 8 & Clade II/group I \\
\hline 14 & OsSTRL14 & LOC_Os08g34330 & $21551327-21549576$ & 8 & Clade II/group I \\
\hline 15 & OsSTRL15 & LOC_Os09g20684 & $12454365-12460996$ & 9 & Clade II/group I \\
\hline 16 & OsSTRL16 & LOC_Os09g20700 & $12466523-12467998$ & 9 & Group II \\
\hline 17 & OsSTRL17 & LOC_Os09g20720 & $12484352-12484831$ & 9 & Clade II/group I \\
\hline 18 & OsSTRL18 & LOC_Os09g20810 & $12536621-12539847$ & 9 & Singleton/group I \\
\hline 19 & OsSTRL19 & LOC_Os10g39710 & $21224367-21226941$ & 10 & Singleton/group I \\
\hline 20 & OsSTRL20 & LOC_Os11g04660 & $1986480-1988225$ & 11 & Clade IV/group I \\
\hline 21 & OsSTRL21 & LOC_Os12g04424 & $1882578-1888289$ & 12 & Clade IV/group I \\
\hline
\end{tabular}

Table 1. The rice OsSTRL genes information. The number designation was based on the position from the top to the bottom on the rice chromosomes 1 to 12 .

A

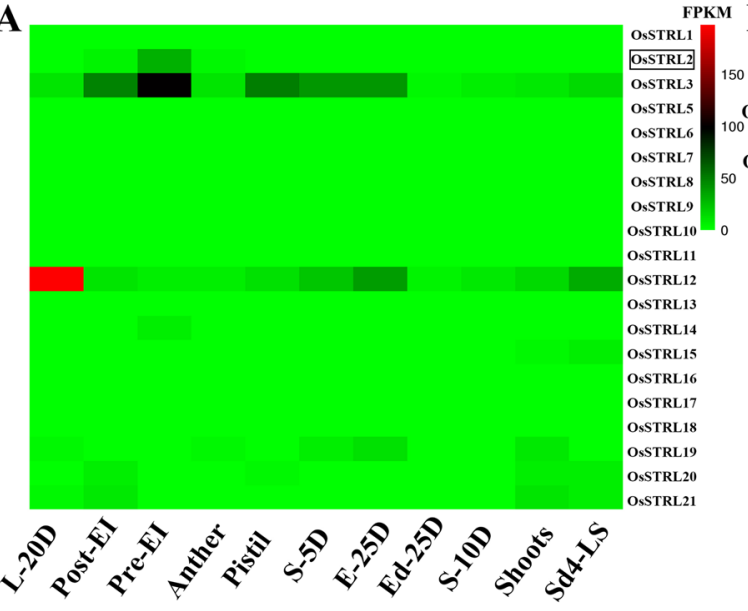

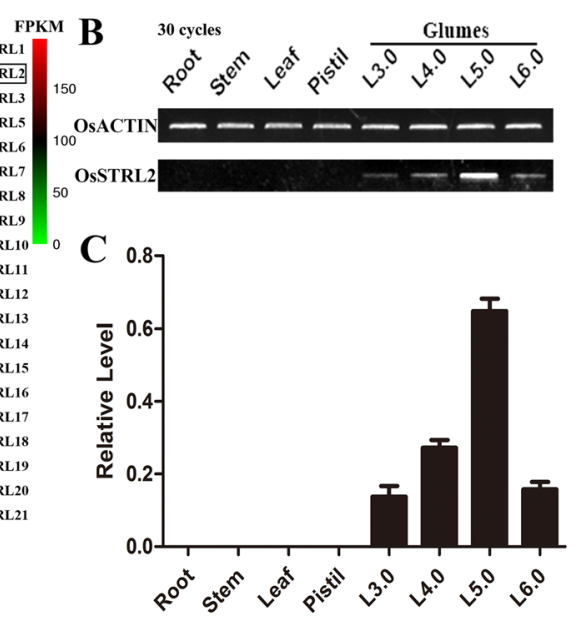

FPKM B 30 cycles

Figure 1. Expression patterns of OsSTRL genes and expression analysis of OsSTRL2. (A) Heat map of OsSTRL members' expression patterns. The absolute FPKM of each OsSTRL gene from NGSTD were used for the heat map analyses. The scale indicates the FPKM expression values. L-20D, leaves at 20 days; Post-EI, postemergency inflorescence; Pre-EI, pre-emergency inflorescence; S-5D, seed at 5 days after pollination; E-25D, Embryo at 25 days after pollination; Ed-25D, endosperm at 25 days after pollination; S-10D, seed at 10 days after pollination; Sd4-LS, seedling at the 4-leaf stage. (B and C) Semi RT-PCR and qPCR for spatial and temporal expression analysis of OsSTRL2. L3.0, glume length of $3.0 \mathrm{~mm}$; L4.0, glume length of $4.0 \mathrm{~mm}$; L5.0, glume length of $5.0 \mathrm{~mm}$; L6.0, glume length of $6.0 \mathrm{~mm}$. The cycles of semi RT-PCR are 30.

because this gene had 0 FPKM expression values in all tissues in NGSTD. Further investigation of the expression profile of OsSTRL members through semi-quantitative real time PCR (semi-RT-PCR, Supplementary Figure 3) showed that OsSTRL19, OsSTRL20, and OsSTRL21 were expressed in all the tissues investigated. OsSTRL5 and OsSTRL6 showed a similar expression pattern of having a specific but weak transcription signal in the young panicles. The expression values of OsSTRL 8 and OsSTRL11 were extremely low in all tissues, although they were expressed in the anthers. OsSTRL14 and OSSTRL15 were expressed in young panicles and in other vegetative organs (leaf, stem, and root). Interestingly, only OsSTRL2 showed a markedly specific transcript signal in both 
A

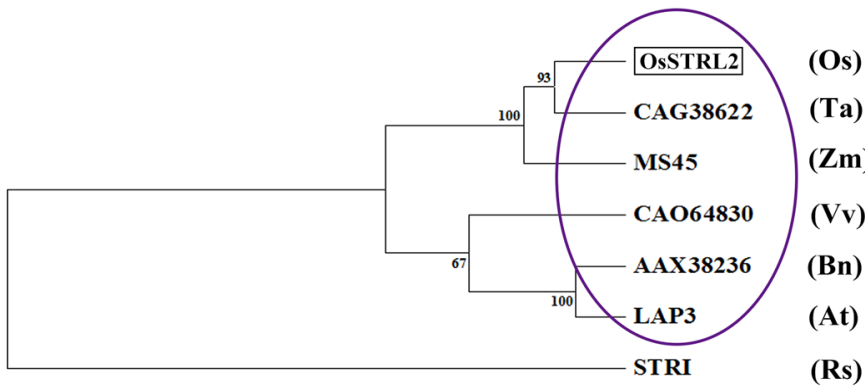

Identities

$(100 \%)$

(82.2\%)

(79.9\%)

(58.8\%)

$(60.5 \%)$

(60.5\%)

$(24.3 \%)$

B
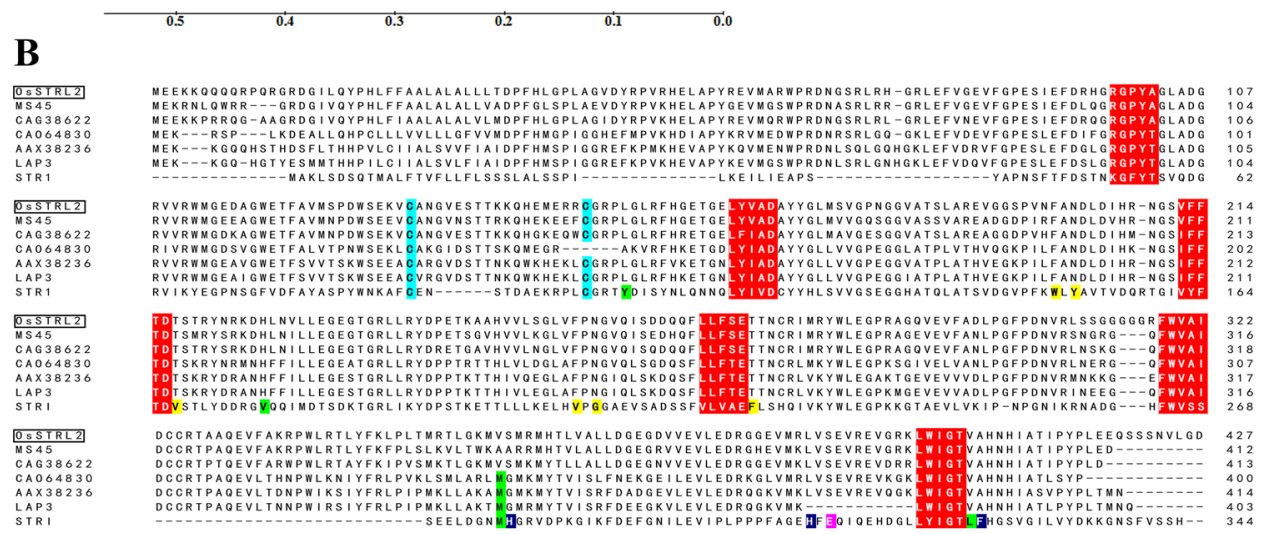

Figure 2. Phylogenetic analysis and alignment of OsSTRL2-related proteins with STR1. (A) MEGA5 program, which used the Neighbor-Joining method with default parameters in addition to 1,000 bootstrap replications to generate the phylogenetic tree of OsSTRL2, OsSTRL2-homologies in different species and STR1. The purple ellipse indicates the genes in the same branch. The numbers at the nodes indicate the bootstrap value. The percentage identities represent the similarities between corresponding protein sequences and OsSTRL2. At, Arabidopsis thaliana; Bn, Brassica napus; Os, Oryza sativa; Rs, Rauvolfia serpentine; Ta, Triticum aestivum; Vv, Vitis vinifera; Zm, Zea mays. (B) Alignment of seven proteins in Fig. 2A using Clustalw2. Red background characters indicate the motifs for $\beta$-propeller folds. Light blue background characters indicate the conserved residues forming the disulfide bridge that pulls two $\alpha$-helices together. Green background characters indicate the residues' contact with the terpenoid part of secologanin. Yellow background characters indicate the residues' contact with the indole part of strictosidine. Deep blue background characters indicate the residues' contact with the glucose moiety. Magenta background character indicates the key catalytic residue of typical strictosidine synthase.

pre-emergency inflorescence, young panicles, and anthers. For further validation, we examined OsSTRL2 expression patterns by using semi-RT-PCR (Fig. 1B) and quantitative real time PCR (q-PCR) (Fig. 1C) in a range of rice organs, including vegetative tissues, namely, roots, stems, and leaves, and reproductive tissues, such as pistils and anthers, at different stages of development. The results showed an exclusive and obvious expression level of OsSTRL2 in the anther with glume lengths of $3.0 \mathrm{~mm}$ to $6.0 \mathrm{~mm}$, indicating that OsSTRL2 might have a function in anther development.

Using the full-length amino acids of OsSTRL2 as a query, we performed a BLASTP search according to the Phytozome database (www.phytozome.net) to explore the potential function of OsSTRL2. Five putative homologs of OsSTRL2 were obtained in Vitis vinifera $(\mathrm{Vv})$, Zea mays $(\mathrm{Zm})$, Arabidopsis thaliana (At), Triticum aestivum (Ta), and Brassica napus (Bn). The homolog amino acid sequences, including STR1 and OsSTRL2, were subsequently aligned with Clustalw $2^{31}$, and a phylogenetic tree was generated from the MEGA $5^{32}$. The phylogenetic tree showed that OsSTRL2 and its related proteins, which were identified by Phytozome BLASTP, were grouped into the same clade, whereas only STR1 belonged to the other branch (Fig. 2A). OsSTRL2 shared $\sim 82.2 \%, \sim 79.9 \%$, $\sim 58.8 \%, \sim 60.5 \%$, and $\sim 60.5 \%$ identities with the OsSTRL2-related protein sequences in Ta, $\mathrm{Zm}, \mathrm{Vv}, \mathrm{Bn}$, and Arabidopsis, respectively (Fig. 2). However, only $24.3 \%$ identity was found between OsSTRL2 and STR1 proteins (Fig. 2). The previous 3D-structural and functional analyses suggested that the six-bladed $\beta$-propeller and three $\alpha$-helices formed the basic structure of the STR1 protein; some important residues were located in the STR1 substrate-binding region, in which the Glu-309 was experimentally proven to be the key catalytic residue ${ }^{4}$. Although the motifs for $\beta$-propeller folds and the residues forming a disulfide bridge that pulls two $\alpha$-helices together were conserved ${ }^{4-7}$, several variations were found within the substrate-binding region in both OsSTRL2 and its homologues of the same branch, including the key site of Glu-309 (Fig. 2B). All OsSTRL members lack the residue Glu-309 which acts as the key catalytic residue of STR1 protein ${ }^{4}$ (Supplementary Figure 1). Moreover, the critical residues that were not found in the OsSTRL2 protein were also absent in the two Arabidopsis strictosidine synthase-like (ATSsl) proteins, ATSsl7 and ATSsl14 (Supplementary Figure 4), which do not exhibit STR 
A

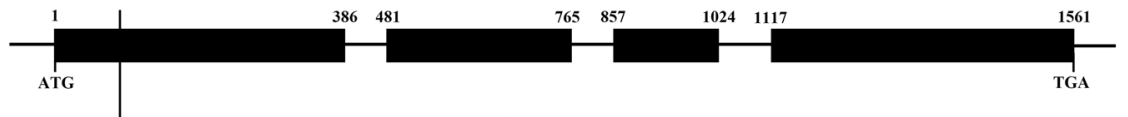

B

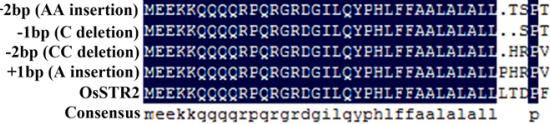

+2bp (AA insertion) STSARSPGWTTGR. . . .

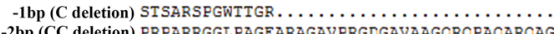
+1bp (A insertion) PRPARRGGLPAGEARAGAVPRGLGAVAAG OsSTR2 2 LRARRGLIFAGEARAGAVRGLGAVAAG $\times$ RCPACARCAG OsSTR2
Consensus

GGACGAGGAGTGGCTGGGCAAGG

CCTGCTCCTCACCGACCCGTTCC

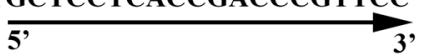

$+2 \mathrm{bp}$ ССТ GCTAACCTCACCGACCCGTTCC -2bp CCTGCT--TCACCGACCCGTTCC -1bp CCTGCT-CTCACCGACCCGTTCC +1 bp CCTGCTACCTCACCGACCCGTTCC

+2 bp (AA insertion) -2bp (CC deletion) VRRRGVRAGVHRVRPPRPRPIRRPRRRPRRAVLGGGRRVG +1bp (A insertion) VRRRGVRAGVHRVRPPRPRPLRR PRRR RRRAVLGGGRRVG OsSTR2 FVGEVFGPESIEEDRHGRGPYAGLADGRVVRWMGEDAGWE
Consensus

+2bp (AA insertion)

-1 bp (C deletion)

-2 bp (CC deletion) DVRRHE.

+1bp (A insertion) LVRRHEP.

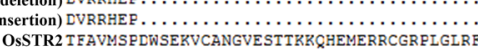
Consensus
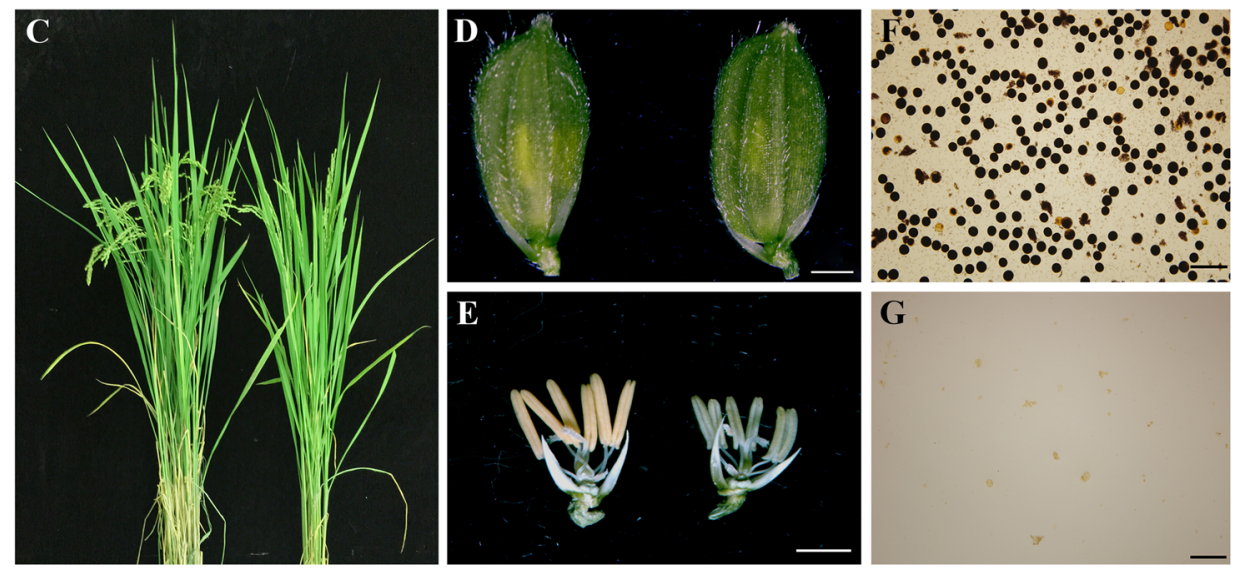

G

Figure 3. Sequence analysis and phenotypic observation of OsSTRL2 CRISPR/Cas9-mediated mutant. (A) Gene structure of OsSTRL2 and mutation analysis of OsSTRL2 gene in transgenic plants. The sequence (5'-CCTGCTCCTCACCGACCCGTTCC-3') located in the first exon of the OsSTRL2 gene was selected as the target site of sgRNA. The black boxes indicate the exons. The red characters indicate the PAM. The green characters indicate the four different types of mutation events generated by CRISPR-Cas9 in the mutants (The detailed information of four different mutation types are listed in Supplementary table S2). (B) Putative amino acid sequences alignment of four types of mutation as described in Fig. 3A. The sequences were aligned using DNA-MAN and displayed using BOXSHADE. The deep blue boxes indicate the $100 \%$ identities of amino acids. (C) Phenotypic comparison of the WT (left) and the mutant plant (right) at the maturity stage. (D) Panicle comparison of the WT (left) and the mutant (right) at the heading stage. (E) Floral comparison of the WT (left) and the mutant (right) (with the palea and lemma removed) at the heading stage. (F and $\mathbf{G}) \mathrm{I}_{2}-\mathrm{KI}$ pollen staining for the WT and the mutant. Bars $=0.20 \mathrm{~mm}(\mathbf{D}$ and $\mathbf{E}) ; 30 \mu \mathrm{m}(\mathbf{F}$ and $\mathbf{G})$.

enzymatic activity ${ }^{33}$. These results suggested that OsSTRL2, which was specifically expressed in the developing anther, might be an atypical strictosidine synthase.

Knockout of OsSTRL2 in rice causes male sterility. The loss-of-function mutations in At Lap3 and Zm Ms45, two putative orthologues of OsSTRL2 (Fig. 2A), resulted in male sterility and defects in the pollen wall formation $^{34,35}$, suggesting that OsSTRL2 might share conserved protein functions with LAP3 and MS45. To investigate the role of OsSTRL2 in rice development, we obtained 29 independent OsSTRL2 knockout plants by using the CRISPR/Cas9-mediated genome-editing tool. The target site in the leaves of $\mathrm{T}_{0}$ transgenic lines were sequenced to determine the mutation of the target sequence (Fig. $3 \mathrm{~A}$ ). Mutations in the target site were detected in 22 out of $29 \mathrm{~T}_{0}$ plants - four plants carried homozygous mutations, 15 plants carried bialleic mutations, and three plants carried heterozygous mutations (Supplementary Table 1). Among these mutants, four different mutation types were identified in the target site (Fig. 3A and Supplementary Table 2), including two adenosine insertions, two cytosine deletions, one cytosine deletion, and one adenosine insertion. These mutations yielded premature stop codons and predictions to produce four types of truncated polypeptides (Fig. 3B and Supplementary Table 2).

No difference was observed between the wild-type plants Oryza sativa japonica cultivated variety Nipponbare (WT) and the $\mathrm{T}_{0}$ plants during vegetative growth (Figs. $3 \mathrm{C}$ and $\mathrm{D}$ ). However, all $\mathrm{T}_{0}$ plants carrying homozygous 

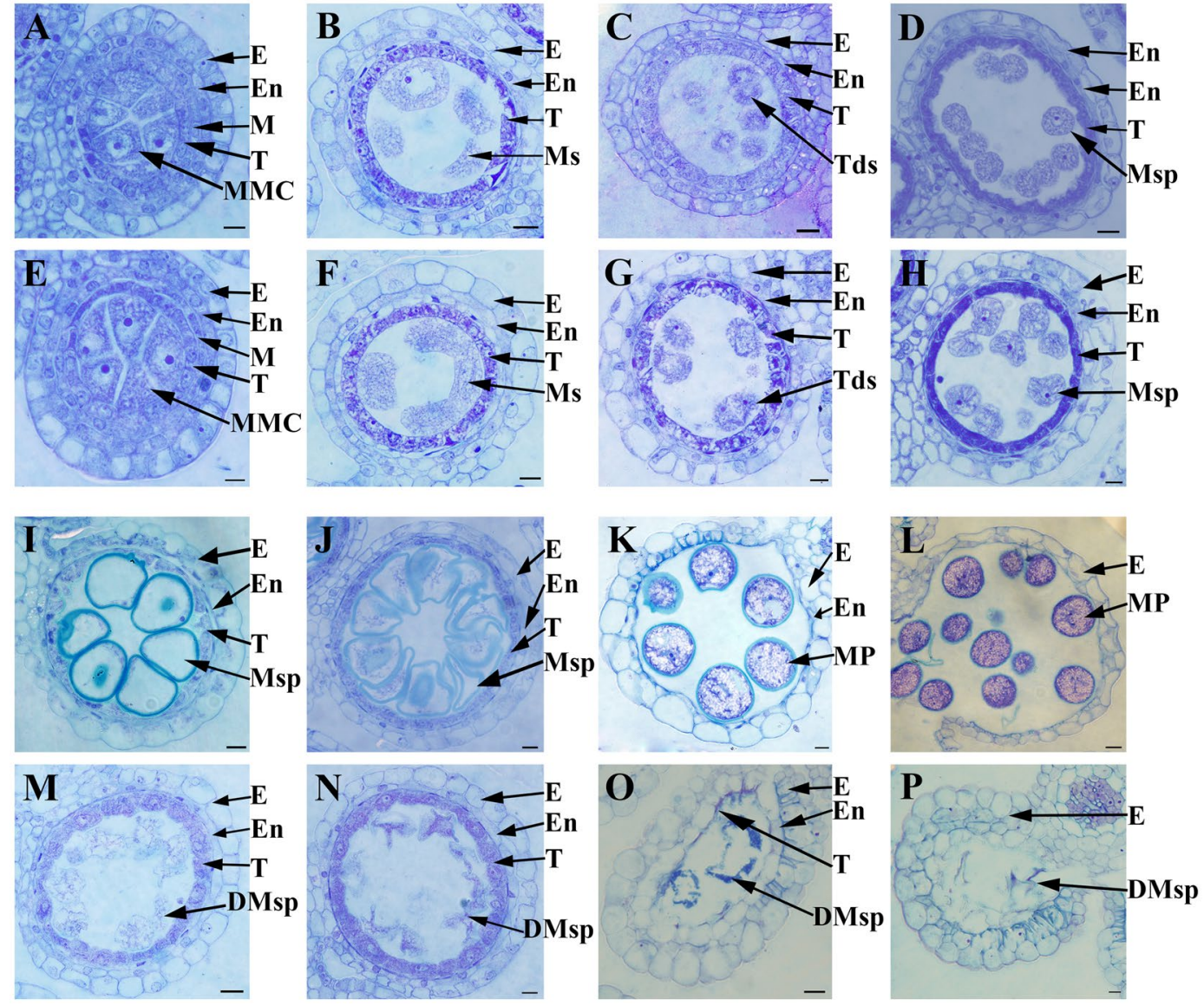

Figure 4. Semi-thin section comparison of anther development between the WT and OsSTRL2 knockout mutant. Comparison of the eight stages of anther development between the WT and the mutant. (A and E) stage 7; (B and F) stage 8a; (C and $\mathbf{G})$ stage $8 \mathrm{~b} ;(\mathbf{D}$ and $\mathbf{H})$ stage 9 ; (I and $\mathbf{M})$ stage 10; ( $\mathbf{J}$ and $\mathbf{N})$ stage 11; (K and $\mathbf{O})$ stage 12; and (L and $\mathbf{P}$ ) stage 13. The WT sections are shown in (A-D and $\mathbf{I}-\mathbf{L})$, and the sections of the mutant are shown in (E-H and $\mathbf{M}-\mathbf{P})$. DMsp, degenerated microspores; E, epidermis; En, endothecium; M, middle layer; MMC, microspore mother cell; MP, mature pollen; Ms, microsporocyte; Msp, microspore; T, tapetum; Tds, tetrads. Bars $=20 \mu \mathrm{m}$.

or biallelic mutations had short whitish anthers with very few pollen grains, which could not be stained by the $\mathrm{I}_{2} / \mathrm{KI}$ solution (Figs. 3E-G). By contrast, all heterozygous $\mathrm{T}_{0}$ plants exhibited normal fertility as observed in the WT (Supplementary Table 1). Similar results were observed in several F2 populations generated by the crosses of these mutants and the WT, thereby further confirming the association of phenotype and genotype. The findings are as follows: (1) the binary construct for targeting the OsSTRL2 site had a high targeted editing efficiency, (2) the phenotype of male sterility in the knockout plants was caused by the mutations revealed in the gene OsSTRL2, and (3) OsSTRL2 played an essential role in controlling male fertility.

Defects of anther wall and pollen exine development in OsSTRL2 knockout lines. We performed transverse section analysis for the anthers of the WT and the mutant to investigate the defects of pollen development in the knockout mutant of OsSTRL2. The previous study classified the rice pollen and anther development into 14 stages from the formation of stamen primordium to the release of mature pollen during anther dehiscence $^{36,37}$. At stage 7, meiosis was initiated by the WT MMC within the locule that was surrounded by the four-layered anther wall of epidermis, endothecium, middle layer, and tapetum from the surface to the interior (Fig. 4A). MMC subsequently formed dyads and tetrads after two steps of meiosis (Figs. 4B and C). Towards the end of stage $8 \mathrm{~b}$, the tapetal cells initiated linearization and centralization, and tapetum had close cell arrangement $^{36,37}$ (Fig. 4C).

No detectable defects were observed between the WT and the mutant anthers until the anther stage 9 (Figs. 4A-C and E-G). However, the mutant anther showed clear morphological differences after stage 8b. At stage 9 , the mutant microspores had a slightly wrinkled shape, although these were released normally as with WT (Figs. 4D and H). In stage 10, the WT microspores were vacuolated and became round-shaped. The tapetal cells started to deteriorate (Fig. 4I), and asymmetric mitosis occurred in vacuolated microspores, which formed generative and vegetative cells at the stage 11 (Fig. 4J). The tapetum of WT gradually thinned from stages 10 to 11(Fig. 4I-J). By contrast, the wrinkled microspores in the mutant had more observable shrinkage deformity from stages 10 to 11 (Figs. 4M,N). The delayed degradation of the tapetum was also observed in the mutant at the 

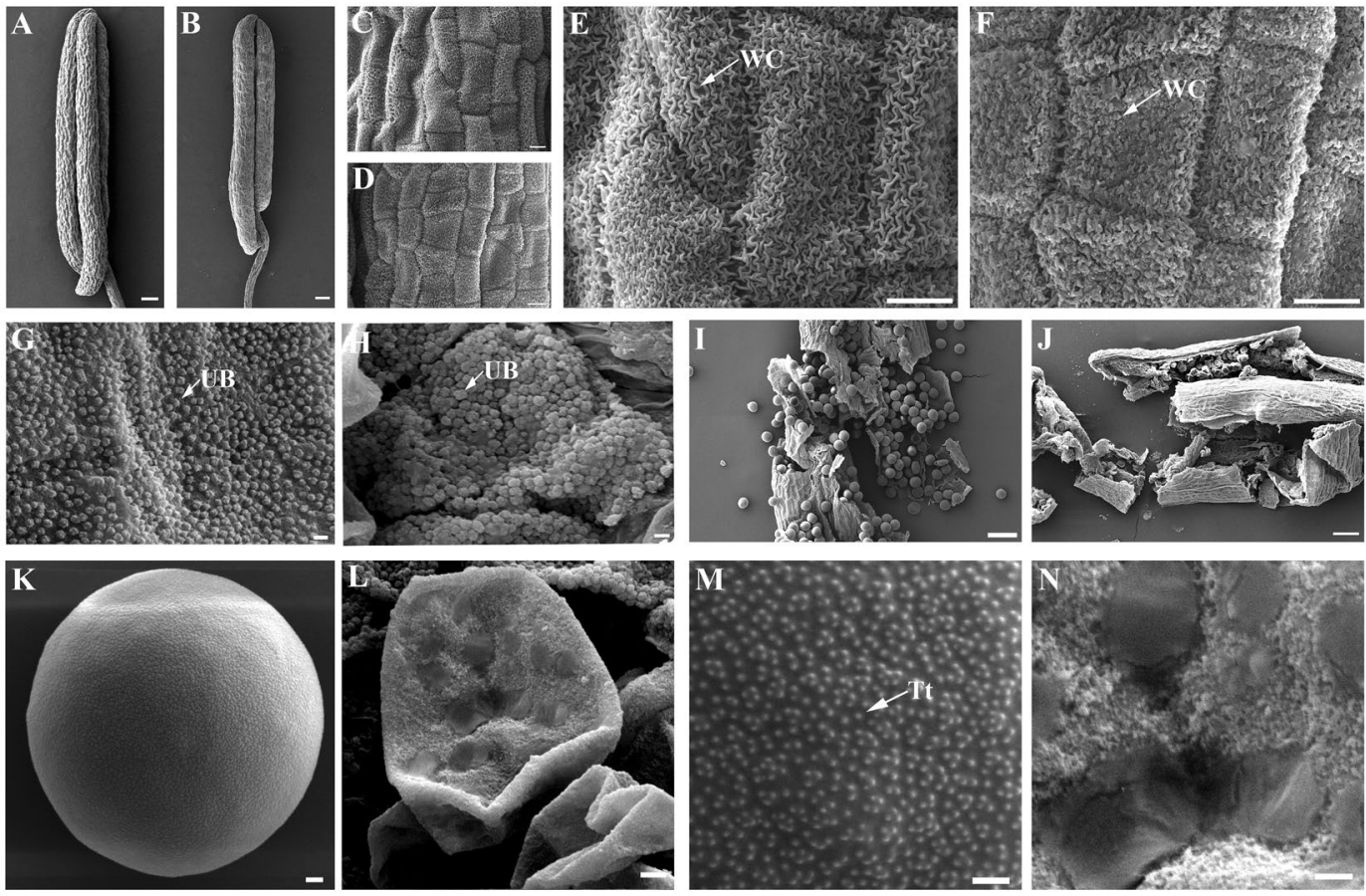

Figure 5. SEM observation for the WT and OsSTRL2 knockout mutant anther and pollen at the stage 12. (A and $\mathbf{B})$ Anthers of the WT and mutant. (C and $\mathbf{D})$ Anther epidermis of the WT and mutant. (E and $\mathbf{F})$ In the enlarged images of epidermal surface of the WT and mutant anthers, the arrows indicate the wax crystal (WC). $(\mathbf{G}$ and $\mathbf{H})$ In the inner surface of the WT and mutant anthers, the arrows indicate the ubisch body (UB). (I and $\mathbf{K})$ Pollen grains in the WT anthers. ( $\mathbf{J}$ and $\mathbf{L}$ ) Pollen grains in the mutant anthers. ( $\mathbf{M}$ and $\mathbf{N}$ ) Outer surface of pollen grains of the WT and mutant; the arrows indicate the tectum (Tt). Bars $=100 \mu \mathrm{m}(\mathbf{A}, \mathbf{B}, \mathbf{I}$ and $\mathbf{J}) ; 10 \mu \mathrm{m}$ $(\mathbf{C}-\mathbf{F}) ; 2 \mu \mathrm{m}$ (K and $\mathbf{L}) ; 1 \mu \mathrm{m}(\mathbf{G}, \mathbf{H}, \mathbf{M}$, and $\mathbf{N})$.

stage 10. In contrast to that in the WT, the mutant anthers still maintained a thick layer of tapetal cells in stage 11 (Figs. 4M,N). At stage 12, the microspores of the WT were round, enlarged, enriched in starch, and developed into mature pollens that filled the anther locule (Fig. 4K). However, the microspores of the mutant were extremely wrinkled, and the inner surface of mutant anther wall still had an incompletely degraded tapetum (Fig. 4O). At stage 13 , the defected microspores of the mutant wrinkled into a distorted and vermiform shape, which thereby emptied the locules of the mutant anthers, whereas the WT anthers were filled with mature pollen and started dehiscence (Figs. 4L and P).

Scanning electron microscopy (SEM) was used during stage 12 anther samples of the WT and the mutant to obtain detailed information on the defects of the anther wall and pollen exine in the mutant. In agreement with the phenotypic observation results (Fig. 3E), the anthers of the mutant (Fig. 5B) were also shorter and smaller than those in the WT (Fig. 5A). In contrast to those with the WT (Figs. 5C and E), the wax crystals of the mutant anthers exo-surface were crowded and less regularly arranged, leading to a compact cell arrangement of anther epidermis (Figs. 5D and F). Orbicules are critical for exporting materials from tapetum to microspores $^{38}$. However, compared with the smooth and regularly arranged orbicules emerging in the WT (Fig. 5G), slightly rough and congested orbicules were formed by the mutant inner locule side of tapetum surface (Fig. $5 \mathrm{H})$. Consistent with the transverse section results (Fig. 4P), the mutant produced less, deflated, and adherent pollen grains (Fig. 5J), whereas the pollen grains in the WT were abundant and round-shaped (Fig. 5I). The exine, mainly composed of sporopollenin, is the most important outer layer of the pollen wall ${ }^{39,40}$. We scanned the surfaces of pollen grains in the WT and the mutant. The pollen grains in the WT showed elaborate exine patterning and roof-like tectum structure on their surface (Figs. $5 \mathrm{~K}$ and M). By contrast, the exine surface was discontinuous and plush-like, and the roof-like tectum structure was generally lost in the mutant (Figs. 5L and N). These results suggested that OsSTRL2 was involved and played an important role in the network of regulating anther wall formation, tapetum degeneration and pollen exine development in rice.

OsSTRL2 was specifically expressed in tapetum and microspores. To directly determine the spatial expression pattern of OSSTRL2, we applied promoter-GUS reporter system to detect its transcriptional activity in the tissues examined with semi-RT-PCR and q-PCR analyses. The transgenic lines containing a GUS-reporter gene driven by the OsSTRL2 promoter further confirmed the specific expression of OsSTRL2 in the developing anther (Figs. 6A-F). Particularly, the section of the GUS-staining anther showed that OsSTRL2 was expressed in the tapetum and microspores (Fig. 6H). RNA in situ hybridization was performed using the WT anther sections to precisely investigate the spatial and temporal expression patterns of OsSTRL2. The results revealed that 

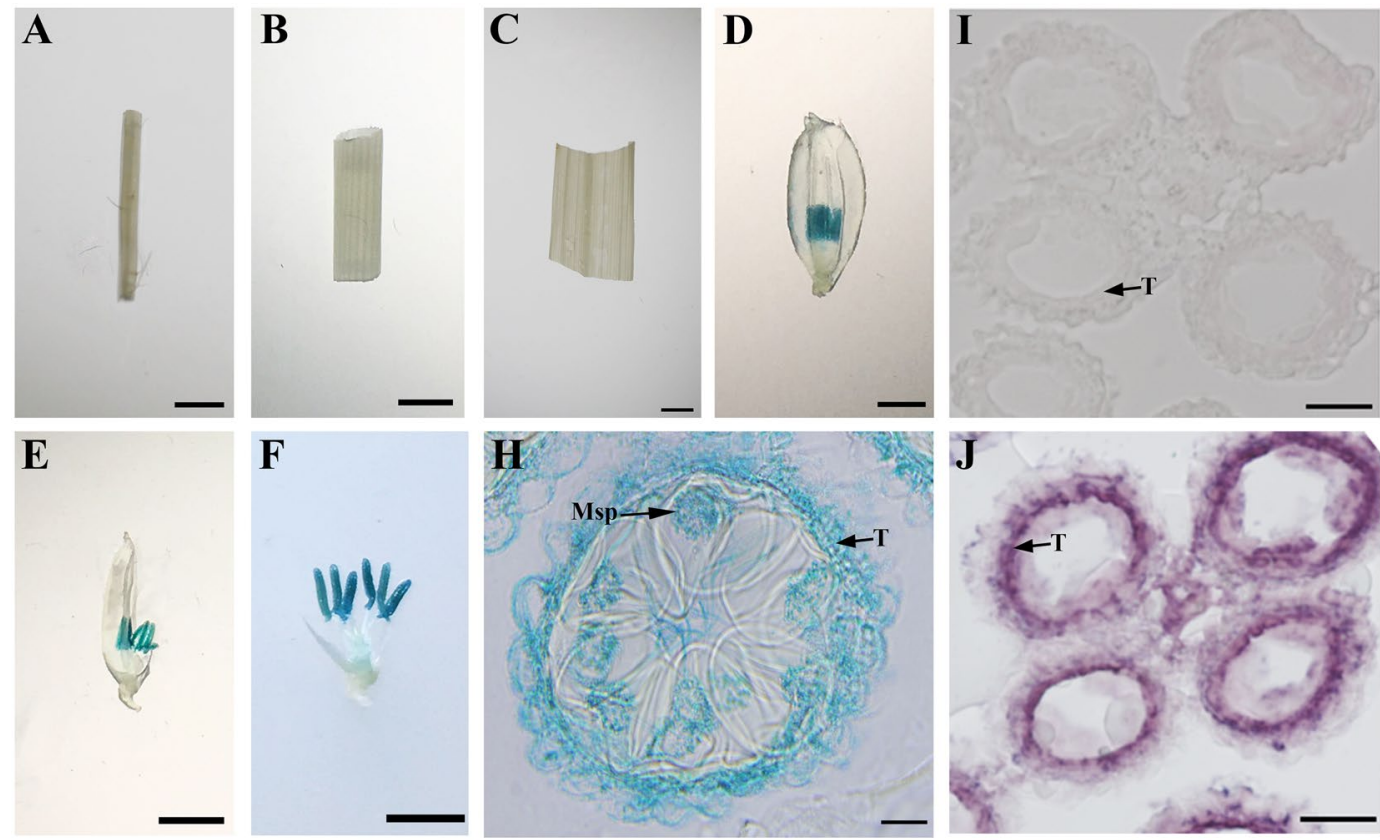

Figure 6. GUS-reporter analysis and RNA in situ hybridization of OsSTRL2. (A-H) GUS expression (blue stained) patterns of root, stem, leaf, floret, spikelet (with the palea removed), spikelet (with the palea and lemma removed) and the section of GUS-staining anther of the OsSTRL2pro::GUS transgenic line, respectively. RNA in situ hybridization using the WT anther sections. (I) Sense probe. (J) Antisense probe. Bars = $3 \mathrm{~mm}(\mathbf{A}-\mathbf{F})$; $30 \mu \mathrm{m}(\mathbf{H}) ; 150 \mu \mathrm{m}(\mathbf{I}$ and $\mathbf{J})$.

OsSTRL2 expression was strong and specifically occurred in the tapetum at stage 8 (Fig. 6J). All these results support the functional role of OsSTRL2 in regulating pollen development.

OsSTRL2 protein is primarily localized to the ER. To determine the subcellular localization of OsSTRL2, we generated a yellow fluorescent protein (YFP) fused to the C-terminal of OsSTRL2, under the control of the cauliflower mosaic virus double 35 S promoter $(2 \times 35 S$ ::OsSTRL2-YFP). Transiently expressed results showed that the YFP signal was localized at the endoplasmic reticulum (ER) that surrounds the nuclei (ER-ring) in the tobacco leaf epidermal cells (Figs. 7B and C) and $2 \times 35$ S:: YFP as the control (Fig. 7A). Moreover, YFP signals were also detected on some tubular and cisternal patterns similar to the ER-like structure (Figs. 7B and C). The co-infiltration of Agrobacterium containing the ER-marker and $2 \times 35 S$ ::OsSTRL2-YFP to the epidermal cell layers of tobacco leaves was conducted to confirm the complex localization of OsSTRL2. Confocal laser-scanning microscopy indicated that the YFP signals (Fig. 7D) were well merged with the ER red fluorescent protein (RFP) signals (Figs. 7E and F). Thus, OsSTRL2 proteins were primarily localized in the ER-ring and in the tubular and cisternal ER structures. These results also suggested that the ER might be an important site for the regulation of pollen exine formation.

\section{Discussion}

Strictosidine is the common precursor of all indole alkaloids and is synthesized from tryptamine and secologanin under STR catalysis, which is the key step of TIA biosynthesis ${ }^{1,3}$. The plant STR is expressed in active meristematic tissues, such as young leaves, flower buds, shoot tips, and petals during plant development, and has a close connection with cell division ${ }^{41}$.

The genome-wide analysis showed that rice contains at least 21 OsSTRL genes that were divided into two groups (Table 1 and Supplementary Figure 2). The large family members of OsSTRL indicated their essential role in rice growth and metabolism. The expression patterns analysis of OsSTRL genes from public data and semi RT-PCR (Fig. 1 and Supplementary Figure 4) showed that five OsSTRL members (OsSTRL3, OsSTRL12, OsSTRL19, OsSTRL20, and OsSTRL21) were constitutively expressed in all tissues, whereas nine members (OsSTRL1, OsSTRL4, OsSTRL7, OsSTRL9, OsSTRL10, OsSTRL13, OsSTRL16, OsSTRL17, and OsSTRL18) displayed extremely weak transcription signals in any of the tissues investigated, which suggests that these genes might be expressed at specific developmental stages or under special conditions. However, only OsSTRL2 had strong and specific transcription signals in pre-emergency inflorescence, young panicles, and anther (Fig. 1A). Similar to male sterility-related genes that were recently identified, OsSTRL2 was preferentially expressed in the developing anther before mature pollen formation as suggested by the additional investigation via semi-RT-PCR and q-PCR (Figs. 1B and C).

Protein sequence alignment analysis reveals that the OsSTRL2 possesses high identities of $79.9 \%$ and $60.5 \%$ of MS45 (maize) and LAP3 (Arabidopsis), respectively (Fig. 2). Both MS45 and LAP3, also annotated in the STR family, are specifically expressed in their anthers, and the mutants of these two genes are male sterile $\mathrm{e}^{34,35}$. In 

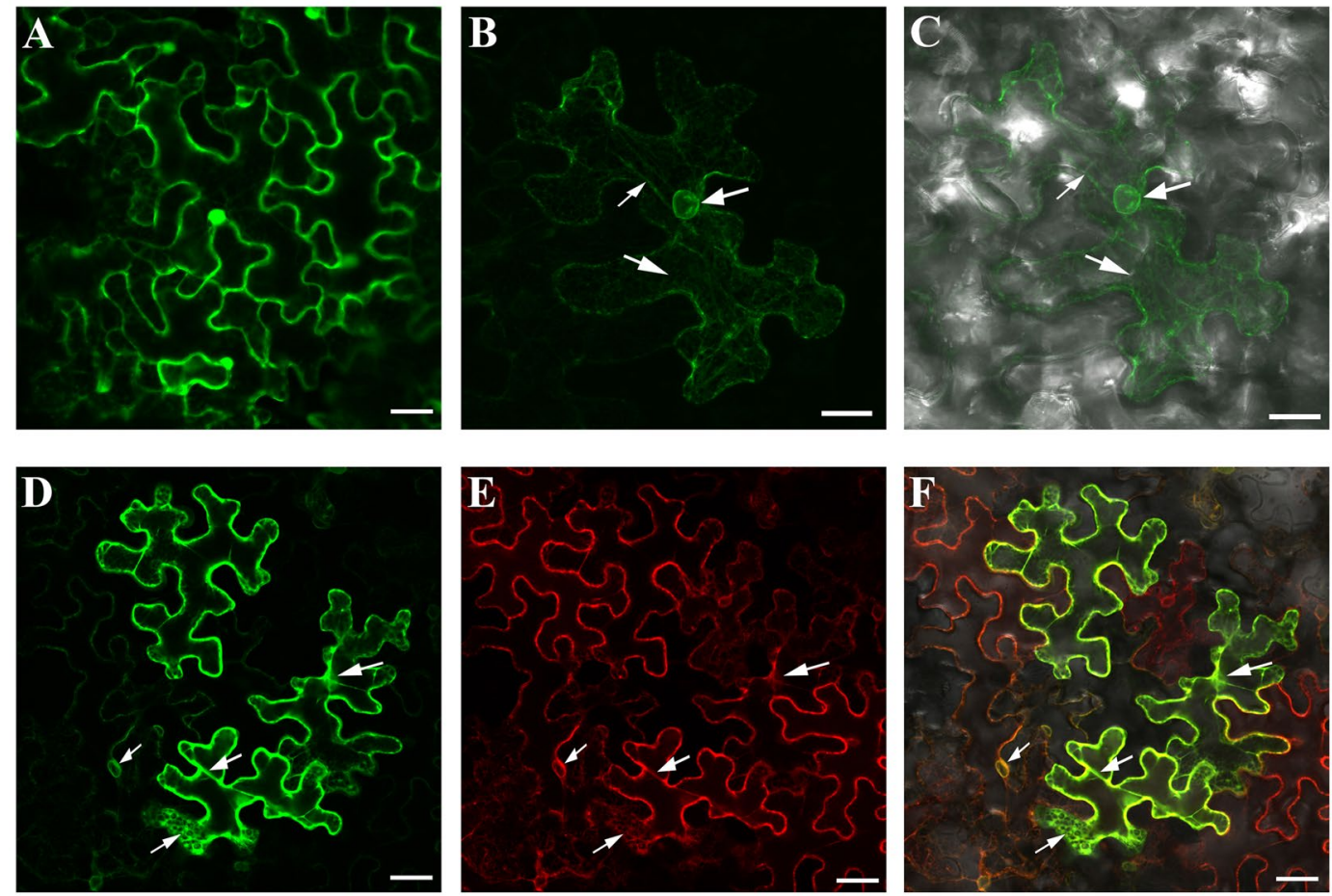

Figure 7. Subcellular localization of OsSTRL2-YFP in tobacco leaf epidermal cells. Confocal images of tobacco leaf epidermis cells after 72 hours of infection were shown. (A) YFP signal of $2 \times 35$ S::YFP as control. (B) YFP signal of $2 \times 35$ S::OsSTRL2-YFP. (C) Merged image of (B) and bright field. (D and E) The YFP and RFP signal of co-expressed $2 \times 35$ S::OsSTRL2-YFP and ER-marker. (F) Merged signal of (D and E) and bright field. Bars $=10 \mu \mathrm{m}(\mathbf{A}, \mathbf{D}, \mathbf{E}$ and $\mathbf{F}) ; 25 \mu \mathrm{m}(\mathbf{B}$ and $\mathbf{C})$.

addition, the knockout of OsSTRL2 in rice causes abnormal male reproductive development phenotypes, including smaller and white anthers and few and defective pollen grains (Figs. 3G, 4P and 5J), suggesting that OsSTRL2 plays vital roles in anther development and pollen formation.

Tapetum, the innermost cell layer of anther wall, produces sporopollenin precursors that are transported to the developing microspores for pollen exine formation ${ }^{42-44}$. Ubisch body, also named orbicule, is located on the inner surface of the tapetal cells and is only a few microns $(\mu \mathrm{m})$ in size ${ }^{45}$. Ubisch body is one of the most essential by-products in pollen wall sporopollenin synthesis ${ }^{46}$. Within the OsSTRL2 knockout mutant anther, tapetum degeneration was delayed (Figs. $4 \mathrm{M}$ and $\mathrm{N}$ ), and the ubisch bodies in the mutant inner locule side of tapetal surface had irregular shape and chaotic arrangement compared with those in the WT (Fig. 5H). Moreover, epidermal waxes can primarily protect the land plants from water loss and can also be involved in plant defenses against pathogens ${ }^{47,48}$. Compared with the WT anther, the crowded permutation and distorted appearance of wax crystals on the mutant outer epidermis, might be the main reason for the smaller anther exo-surface cell size in the mutant anther (Figs. 5C-F). These results indicated that OsSTRL2 has a key function in the biological pathway of tapetum PCD and ubisch body formation. OsSTRL2 loss-function might affect the expansion and morphology of the anther epidermal cells by producing abnormal wax crystals.

Pollen wall is a surrounded lipidic structure of male gametophyte that plays an essential role in protecting pollen from various environmental stress and bacterial attack. Pollen wall consists of two layers: the outer exine, which contains tectum and baculum; and the inner intine ${ }^{39,40,49,50}$. In Arabidopsis, PKSA and PKSB, two plant type III polyketide synthases (PKSs), mediate the biochemical reactions to induce the synthesis of pollen fatty acids and phenolics found in exine ${ }^{51,52}$. As an Acyl-CoA synthetase catalyzing the fatty acyl-CoA ester biosynthesis reaction in Arabidopsis tapetal cells, ACOS5 plays a central role in generating sporopollenin monomers that can be exported to microspores and regulate the formation of pollen exine ${ }^{53,54}$. LAP3 is a homolog of OsSTRL2 in Arabidopsis (Fig. 2). Lap3 mutant plants produce less adherent pollen grains because of their thinner pollen exine walls $^{34}$. Similar to that in lap3, the OsSTRL2 knockout mutant pollen grains displayed structurally weakened exine that lacks tectum (Fig. 5N). This defect might cause pollen grains to rupture easily and cause adhesion (Figs. 3G, $4 \mathrm{M}-\mathrm{P}$ and 5J). By contrast, the pollen grains in WT were round and arranged by normal roof-like tectum on their outer exine surface (Fig. 5M). These results suggested that OsSTRL2 might use similar regulatory pathways for pollen exine formation in Arabidopsis.

The mutation in Ms45 gene, a maize homolog of OsSTRL2 (Fig. 2), causes the absence of pollen. Immunological analyses showed that MS45 was localized to the tapetal cells during microsporogenesis ${ }^{35}$. Our results indicated that OSSTRL2 was specifically expressed in tapetum and microspores via GUS reporter analysis and RNA in situ hybridization (Fig. 6), suggesting that OsSTRL2 and MS45 might share this tapetal localization and play the conserved roles during exine biosynthesis. PKSA, PKSB, and TKPR1 are required for sporopollenin biosynthesis in 


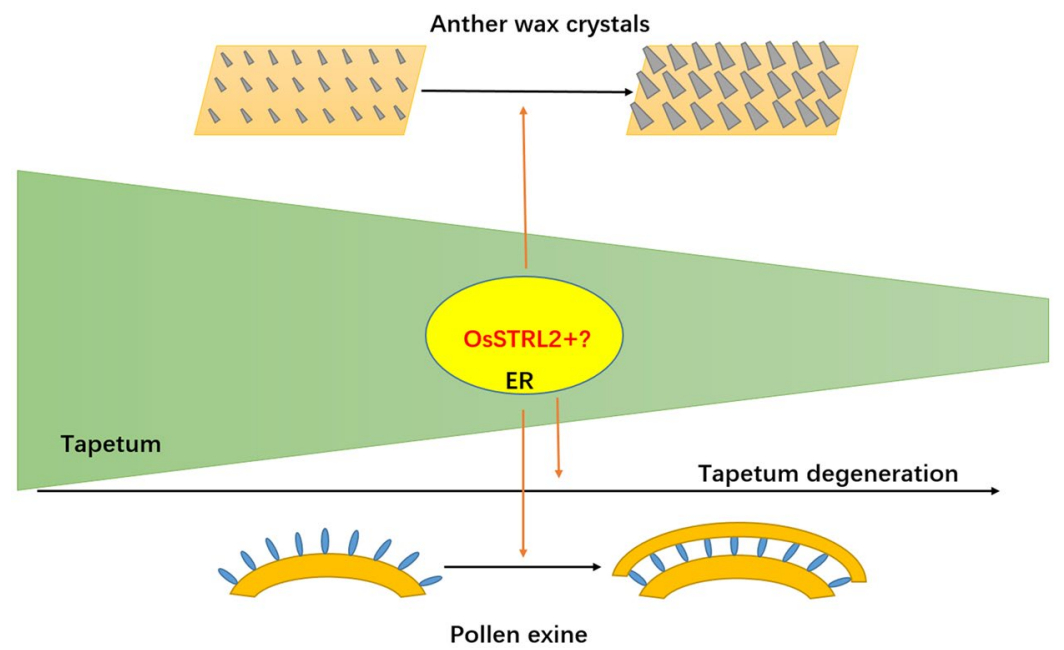

Figure 8. Model for the role of OsSTRL2 during tapetum degeneration, anther development and pollen exine formation. The product generated by OsSTRL2 is involved in the maturation of anther wax crystals, PCD of tapetal cells, and formation of pollen exine.

Arabidopsis ${ }^{51,52,55}$. These enzymes are immunolocalized to the ER of anther tapetal cells ${ }^{56}$. Similarly, our results also showed that OsSTRL2 was mainly localized in the ER (Fig. 7), whereas the STR1 orthologue in periwinkle was simultaneously targeted to the chloroplast, vacuole and ER in transgenic tobacco ${ }^{57}$. These results suggested that the ER of tapetum might be an important site for the proteins regulating pollen wall formation, and therefore OsSTRL2 might participate in this essential network by influencing the tapetum degeneration, anther wax crystal development and pollen exine formation (Fig. 8).

The structural characterizations of STR1 deciphered the 3D structure of the active site of strictosidine synthase and the details of its reaction mechanism ${ }^{3,4,7}$. The structure of STR 1 contains six-bladed $\beta$-propellers and three $\alpha$-helices. The substrate-binding pocket of STR1, in which several vital residues (Fig. 2B) are involved, links the active center to the surface of the substrate molecule. In addition, the structural analysis and site-directed mutagenesis experiments found that Glu-309 (Fig. 2B) in the residues of STR1 binding pocket is a key catalytic residue $^{4,7}$. Among the typical STR family, these motifs and residues are important and conserved because they provide the basic structure of the protein 7 . However, OsSTRL2 lacks Glu-309, which is the key catalytic residue of typical strictosidine synthase STR1. OsSTRL2 also exhibits differences in some residues in the substrate-binding pocket (Fig. 2B), although it contains the motifs for $\beta$-propeller folds and the residues Cys- 89 and Cys-101, which form a disulfide bridge and pull two out of three $\alpha$-helices together (Fig. 2B). This finding suggested the possibility that OsSTRL2 might have no or have low catalytic activity as presented in the typical strictosidine synthase. Similar to OsSTRL2, AtSsl7 and AtSsl14 are Arabidopsis strictosidine synthase-like proteins that lack the Glu-309 and some other residues in the substrate-binding region (Supplementary Figure 4) and do not have strictosidine synthase activity ${ }^{33}$. This result might offer experimental evidence to our hypothesis on the catalytic activity of OsSTRL2.

LAP3 is the homologous gene of OsSTRL2 in Arabidopsis (Fig. 2) and is one of the ATSsl genes ${ }^{4}$. LAP3 has a relatively low similarity to the protein sequence of STR $1^{34}$. Our results also revealed a comparatively low amino acid sequence identity between OsSTRL2 and STR1 (Fig. 2). These findings indicated that both LAP3 and OsSTRL2 have evolutionary divergence with STR1. In addition, Kibble et al. found that all members of the ATSsl family lack the Glu-309 and suggested that this family might not have strictosidine synthase enzyme activity ${ }^{4}$. The absence of this critical catalytic residue also occurs in all OsSTRL family members of rice (Supplementary Figure 1). These differences might bring variations in the substrate specificity and/or enzymatic activity for OsSTRL family members, as well as the possibilities that those different members might participate in other biochemical pathways in plants ${ }^{11}$. Although OsSTRL2 lacks some the key residues of STR, we propose that OsSTRL2 is an atypical strictosidine synthase because it processes the basic framework of a STR protein (e.g., $\beta$-propeller folds and $\alpha$-helices).

In summary, this study identified 21 rice OsSTRL genes through genome-wide analysis. The 21 OsSTRL members were clustered into two major groups and four clades in group I. The expression pattern analysis showed that only OsSTRL2 was specifically expressed in the developing anther. OsSTRL2 loss-function in rice caused the detention of tapetum degeneration, abnormal wax crystals, and orbicules of anther and the absence of pollen exine tectum, which led to the knockout mutant male sterility. Based on the accumulation of OsSTRL2 in tapetal cells and microspores at the transcript level and the ER localization of OsSTRL2-YFP protein, OsSTRL2 might participate in the regulation network of pollen wall formation that occurs in the ER of tapetum. By combining the results of protein sequence alignment, we consider OsSTRL2 as a rice atypical strictosidine synthase that play key roles in anther development and pollen wall formation. Our work also provides a new perception into mechanisms of anther and pollen developmental processes and enhances our understanding of the regulation of pollen exine formation. 


\begin{abstract}
Materials and Methods
Plant materials and growth conditions. All rice (Oryza sativa) plants used in this study were naturally grown and maintained in the experimental field of the Rice Research Institute, Sichuan Agricultural University, Wenjiang, China.
\end{abstract}

Genome-wide sequence analysis of rice STR-like genes and phylogenetic analysis. Using the full-length STR1 protein sequence in Rauvolfia Serpentine as a query, a BLASTP search of the japonica-type rice genome (RGAP; http://rice.plantbiology.msu.edu/) ${ }^{28}$ was performed to forecast the 21 OsSTRL candidates. The amino acid sequences of these candidates were subsequently used for the SMART search ${ }^{29}, 58$ and were aligned by using Clustal W software (www.ebi.ac.uk/Clustalw) to further confirm the accuracy. The OsSTRL2-related protein sequences in different species were identified by Phytozome Blast (https://phytozome.jgi.doe.gov) with default parameters using the full-length amino acid of OsSTRL2 as a query, and the sequences aligned with Clustalw $2^{31}$. In addition to the 1,000 bootstrap replications, the MEGA5 program used the NJ method with default parameters to generate all the phylogenetic trees ${ }^{32}$.

CRISPR/Cas9-mediated mutation and phenotype association assay. To verify the OsSTRL2 function, we generated a gRNA construction, wherein the gRNA and the plant-optimized Cas9 were driven by the rice U3 and maize UBI promoter, respectively ${ }^{59}$. Plasmid was introduced into the WT (Nipponbare). The DNA isolated from the leaves of transgenic plants was subjected to PCR and sequencing analysis with the primer set OsSTRL2-gRNA-seq (see Supplementary Table S3) to evaluate whether the mutation occurred. To verify the association between the candidate mutation site in OsSTRL 2 and the male sterile phenotype, we observed the target site sequence of all the CRISPR/Cas9 transgenic plants via direct or cloned sequencing of the PCR products, which were amplified using the primer set OsSTRL2-gRNA-seq (statistical results are shown in Supplementary Table 1). The co-segregation results were further confirmed in several F2 populations that were generated by backcrossing these mutants with the WT.

Phenotypic characterization. Transverse sections of the anther development analysis were performed as described previously ${ }^{19}$. For the SEM analysis of anther and pollen, the samples at stage 12 were prepared as described by Qin et al. ${ }^{25}$ and then examined with a JSM-7500F scanning electron microscope.

Expression analysis. Digital expression pattern analysis of 21 OsSTRL members was performed by using the public RNAseq data from NGSTD (http://rice.plantbiology.msu.edu/expression.shtml) to draw a heat map with the OmicShare tools, a free online platform for data analysis (http://www.omicshare.com/tools/). Total RNAs from a variety of rice tissues were extracted using the TriPure isolation reagent (Roche, Indianapolis, USA). cDNAs were reverse-transcribed using the Transcriptor First-Strand cDNA Synthesis Kit (Roche, Indianapolis, USA). Semi-RT-PCR was performed with the program involving pre-denaturation at $95^{\circ} \mathrm{C}$ for $2 \mathrm{~min}, 29$ or 30 cycles of the reaction at $95^{\circ} \mathrm{C}$ for $30 \mathrm{~s}, 57^{\circ} \mathrm{C}$ for $30 \mathrm{~s}$ and $72^{\circ} \mathrm{C}$ for $30 \mathrm{~s}$. Another step of at $72^{\circ} \mathrm{C}$ for $7 \mathrm{~min}$ was conducted for the final extension, and the PCR amplification results were run on 1\% agarose gel. The gel image was obtained by using Molecular Image Gel Doc XR + image analysis system (Bio-Rad, Hercules, CA). qPCR experiments conducted by using a Bio-Rad CFX96 real-time PCR System (California, USA) as described by Li et al. ${ }^{60}$, the rice actin gene was used as the internal control, and the fold change for gene expression was calculated as described previously ${ }^{61}$. All primers used in this study are listed in Supplementary Table 3.

Promoter-GUS reporter assays and RNA in situ hybridization. For the transcript level tissue localization of OsSTRL2, a construct of OsSTRL2pro::GUS was generated through pCAMBIA1300 wherein the native OsSTRL2 promoter drove the GUS gene. The OsSTRL2pro::GUS construct was introduced into the Agrobacterium tumefaciens strain EHA105 and then transferred into the WT. Histochemical GUS staining was performed as described previously ${ }^{62}$. A 406 bp fragment of OsSTRL 2 cDNA was amplified using specific primers (Supplementary Table 3) and cloned into pBluescript-SK vector (Stratagene) to generate the OsSTRL2-specific antisense probe. The fragment was transcribed with T7 RNA polymerase. In situ hybridizations including tissue embedding, hybridization and signal detection were conducted as described by Chen et al. ${ }^{63}$.

Subcellular localization of OsSTRL2. The full-length cDNA of OsSTRL2 was cloned into pA7-YFP vector to generate a $2 \times 35 S$ ::OsSTRL2-YFP cassette which fused the C-terminal of OsSTRL2 with YFP under the control of the cauliflower mosaic virus double $35 \mathrm{~S}$ promoter. The entire $2 \times 35 \mathrm{~S}:$ :OsSTRL2-YFP cassette was then inserted into the pCAMBIA1300. The RFP with the C-terminal extension-KDEL served as the ER marker under the control of the constitutive cauliflower mosaic virus double 35 Spromoter ${ }^{64}$. These plasmids were individually expressed or co-expressed in tobacco leaf epidermis cells by agrobacterium-mediated infiltration. YFP and RFP signals were visualized with a confocal scanning microscope (Nikon A1, Kanagawa, Japan) $72 \mathrm{~h}$ after infiltration.

\title{
References
}

1. Yamazaki, Y. et al. Metabolite profiling of alkaloids and strictosidine synthase activity in camptothecin producing plants. Phytochemistry 62, 461-470, doi:10.1016/S0031-9422(02)00543-5 (2003).

2. Kutchan, T. M. Alkaloid Biosynthesis[mdash]The Basis for Metabolic Engineering of Medicinal Plants. The Plant cell 7, 1059-1070 (1995).

3. Smith, G. N. Strictosidine: a key intermediate in the biogenesis of indole alkaloids. Chem. Commun. 912-914, doi:10.1039/ C19680000912 (1968).

4. Stockigt, J., Barleben, L., Panjikar, S. \& Loris, E. A. 3D-Structure and function of strictosidine synthase-the key enzyme of monoterpenoid indole alkaloid biosynthesis. Plant physiology and biochemistry: PPB 46, 340-355, doi:10.1016/j.plaphy.2007.12.011 (2008).

5. Kutchan, T. M. Expression of enzymatically active cloned strictosidine synthase from the higher plant Rauvolfia serpentina in Escherichia coli. FEBS letters 257, 127-130 (1989). 
6. Kutchan, T. M., Hampp, N., Lottspeich, F., Beyreuther, K. \& Zenk, M. H. The cDNA clone for strictosidine synthase from Rauvolfia serpentina. DNA sequence determination and expression in Escherichia coli. FEBS letters 237, 40-44 (1988).

7. Ma, X., Panjikar, S., Koepke, J., Loris, E. \& Stockigt, J. The structure of Rauvolfia serpentina strictosidine synthase is a novel sixbladed beta-propeller fold in plant proteins. The Plant cell 18, 907-920, doi:10.1105/tpc.105.038018 (2006).

8. Shelton, A. L. Variable chemical defences in plants and their effects on herbivore behaviour. Evolutionary Ecology Research 2, 231-249 (2000)

9. Kutchan, T. M. Ecological Arsenal and Developmental Dispatcher. The Paradigm of Secondary Metabolism. Plant physiology 125, 58-60 (2001)

10. Sohani, M. M., Schenk, P. M., Schultz, C. J. \& Schmidt, O. Phylogenetic and transcriptional analysis of a strictosidine synthase-like gene family in Arabidopsis thaliana reveals involvement in plant defence responses. Plant biology 11, 105-117 (2009)

11. Fabbri, M., Delp, G., Schmidt, O. \& Theopold, U. Animal and plant members of a gene family with similarity to alkaloid-synthesizing enzymes. Biochemical \& Biophysical Research Communications 271, 191-196 (2000).

12. Liu, W., Liu, C., Hu, X., Yang, J. \& Zheng, L. Application of terahertz spectroscopy imaging for discrimination of transgenic rice seeds with chemometrics. Food chemistry 210, 415-421, doi:10.1016/j.foodchem.2016.04.117 (2016)

13. Matsumoto, AI, T. E., Shaw, J. F., Al, E. \& Sugiura, M. (Nature, 274: 337-345)The map-based sequence of the rice genome. (2005).

14. Goldberg, R. B., Beals, T. P. \& Sanders, P. M. Anther development: basic principles and practical applications. The Plant cell 5, 1217-1229, doi:10.1105/tpc.5.10.1217 (1993).

15. Sanders, P. M. et al. Anther developmental defects in Arabidopsis thaliana male-sterile mutants. Sexual plant reproduction 11, 297-322, doi:10.1007/s004970050158 (1999).

16. Wilson, Z. A., Morroll, S. M., Dawson, J., Swarup, R. \& Tighe, P. J. The Arabidopsis MALE STERILITY1 (MS1) gene is a transcriptional regulator of male gametogenesis, with homology to the PHD-finger family of transcription factors. The Plant journal: for cell and molecular biology 28, 27-39 (2001).

17. Niu, N. et al. EAT1 promotes tapetal cell death by regulating aspartic proteases during male reproductive development in rice. Nature communications 4, 1445, doi:10.1038/ncomms2396 (2013).

18. Jung, K. H. et al. Rice Undeveloped Tapetum1 is a major regulator of early tapetum development. The Plant cell 17, 2705-2722, doi:10.1105/tpc.105.034090 (2005).

19. Li, N. et al. The rice tapetum degeneration retardation gene is required for tapetum degradation and anther development. The Plant cell 18, 2999-3014, doi:10.1105/tpc.106.044107 (2006).

20. Li, H. et al. PERSISTENT TAPETAL CELL1 encodes a PHD-finger protein that is required for tapetal cell death and pollen development in rice. Plant physiology 156, 615-630, doi:10.1104/pp.111.175760 (2011).

21. Yang, X. et al. Rice CYP703A3, a cytochrome P450 hydroxylase, is essential for development of anther cuticle and pollen exine. Journal of integrative plant biology 56, 979-994, doi:10.1111/jipb.12212 (2014).

22. Li, H. et al. Cytochrome P450 family member CYP704B2 catalyzes the \{omega\}-hydroxylation of fatty acids and is required for anther cutin biosynthesis and pollen exine formation in rice. The Plant cell 22, 173-190, doi:10.1105/tpc.109.070326 (2010).

23. Wang, W. et al. Crystallization and preliminary crystallographic analysis of defective pollen wall (DPW) protein from Oryza sativa. Acta crystallographica. Section F, Structural biology communications 70, 758-760, doi:10.1107/S2053230X14008486 (2014).

24. Shi, J. et al. Defective pollen wall is required for anther and microspore development in rice and encodes a fatty acyl carrier protein reductase. The Plant cell 23, 2225-2246, doi:10.1105/tpc.111.087528 (2011).

25. Qin, P. et al. ABCG15 encodes an $\mathrm{ABC}$ transporter protein, and is essential for post-meiotic anther and pollen exine development in rice. Plant \& cell physiology 54, 138-154, doi:10.1093/pcp/pcs162 (2013).

26. Ko, S. S. et al. The bHLH142 Transcription Factor Coordinates with TDR1 to Modulate the Expression of EAT1 and Regulate Pollen Development in Rice. The Plant cell 26, 2486-2504, doi:10.1105/tpc.114.126292 (2014).

27. Fu, Z. et al. The Rice Basic Helix-Loop-Helix Transcription Factor TDR INTERACTING PROTEIN2 Is a Central Switch in Early Anther Development. The Plant cell 26, 1512-1524, doi:10.1105/tpc.114.123745 (2014).

28. Goff, S. A. et al. A draft sequence of the rice genome (Oryza sativa L. ssp. japonica). Science 296, 92-100, doi:10.1126/science.1068275 (2002).

29. Letunic, I. et al. SMART 4.0: towards genomic data integration. Nucleic acids research 32, D142-144, doi:10.1093/nar/gkh088 (2004).

30. Bailey, T. L. \& Gribskov, M. Combining evidence using p-values: application to sequence homology searches. Bioinformatics 14, $48-54$ (1998)

31. Goujon, M. et al. A new bioinformatics analysis tools framework at EMBL-EBI. Nucleic acids research 38, W695-699, doi:10.1093/ nar/gkq313 (2010).

32. Tamura, K. et al. MEGA5: molecular evolutionary genetics analysis using maximum likelihood, evolutionary distance, and maximum parsimony methods. Molecular biology and evolution 28, 2731-2739, doi:10.1093/molbev/msr121 (2011).

33. Natalieaj, K. et al. Phylogenetic analysis and functional characterisation of strictosidine synthase-like genes in Arabidopsis thaliana. Functional Plant Biology 36, 1098-1109 (2009).

34. Dobritsa, A. A. et al. LAP3, a novel plant protein required for pollen development, is essential for proper exine formation. Sexual plant reproduction 22, 167-177, doi:10.1007/s00497-009-0101-8 (2009).

35. Cigan, A. M., Unger, E., Xu, R. J., Kendall, T. \& Fox, T. W. Phenotypic complementation of ms45 maize requires tapetal expression of MS45. Plant reproduction 14, 135-142 (2001).

36. Zhang, D., Luo, X. \& Zhu, L. Cytological analysis and genetic control of rice anther development. Journal of genetics and genomics $=Y i$ chuan xue bao 38, 379-390, doi:10.1016/j.jgg.2011.08.001 (2011).

37. Zhang, D. \& Wilson, Z. A. Stamen specification and anther development in rice. Chinese Science Bulletin 54, 2342-2353, doi:10.1007/ s11434-009-0348-3 (2009).

38. Wang, A., Xia, Q., Xie, W., Datla, R. \& Selvaraj, G. The classical Ubisch bodies carry a sporophytically produced structural protein (RAFTIN) that is essential for pollen development. Proceedings of the National Academy of Sciences of the United States of America 100, 14487-14492, doi:10.1073/pnas.2231254100 (2003).

39. Blackmore, S., Wortley, A. H., Skvarla, J. J. \& Rowley, J. R. Pollen wall development in flowering plants. The New phytologist 174, 483-498, doi:10.1111/j.1469-8137.2007.02060.x (2007).

40. Edlund, A. F., Swanson, R. \& Preuss, D. Pollen and stigma structure and function: the role of diversity in pollination. The Plant cell 16(Suppl), S84-97, doi:10.1105/tpc.015800 (2004).

41. St-Pierre, B. et al. Multicellular compartmentation of catharanthus roseus alkaloid biosynthesis predicts intercellular translocation of a pathway intermediate. The Plant cell 11, 887-900 (1999).

42. Lallemand, B., Erhardt, M., Heitz, T. \& Legrand, M. Sporopollenin biosynthetic enzymes interact and constitute a metabolon localized to the endoplasmic reticulum of tapetum cells. Plant physiology 162, 616-625, doi:10.1104/pp.112.213124 (2013).

43. Quilichini, T. D., Douglas, C. J. \& Samuels, A. L. New views of tapetum ultrastructure and pollen exine development in Arabidopsis thaliana. Annals of botany 114, 1189-1201, doi:10.1093/aob/mcu042 (2014).

44. Zhang, D. \& Yang, L. Specification of tapetum and microsporocyte cells within the anther. Current opinion in plant biology 17, 49-55, doi:10.1016/j.pbi.2013.11.001 (2014).

45. Kreunen, S. S. \& Osborn, J. M. Pollen and anther development in Nelumbo (Nelumbonaceae). American Journal of Botany 86, $1662-1676(1999)$. 
46. Verstraete B. et al. Orbicules in Flowering Plants: A Phylogenetic Perspective on their Form and Function. Botanical Review. 80(2), $107-134$ (2014).

47. Riederer, M. \& Schreiber, L. Protecting against water loss: analysis of the barrier properties of plant cuticles. Journal of experimental botany 52, 205-208 (2001).

48. Jenks, M. A. et al. Chemically Induced Cuticle Mutation Affecting Epidermal Conductance to Water Vapor and Disease Susceptibility in Sorghum bicolor (L.) Moench. Plant physiology 105, 1239 (1994)

49. Ariizumi, T. \& Toriyama, K. Genetic regulation of sporopollenin synthesis and pollen exine development. Annual review of plant biology 62, 437-460, doi:10.1146/annurev-arplant-042809-112312 (2011).

50. Quilichini, T. D., Grienenberger, E. \& Douglas, C. J. The biosynthesis, composition and assembly of the outer pollen wall: A tough case to crack. Phytochemistry 113, 170-182, doi:10.1016/j.phytochem.2014.05.002 (2015).

51. Dobritsa, A. A. et al. LAP5 and LAP6 encode anther-specific proteins with similarity to chalcone synthase essential for pollen exine development in Arabidopsis. Plant physiology 153, 937-955, doi:10.1104/pp.110.157446 (2010).

52. Kim, S. S. et al. LAP6/POLYKETIDE SYNTHASE A and LAP5/POLYKETIDE SYNTHASE B encode hydroxyalkyl alpha-pyrone synthases required for pollen development and sporopollenin biosynthesis in Arabidopsis thaliana. The Plant cell 22, 4045-4066, doi:10.1105/tpc.110.080028 (2010).

53. Jiang, J., Zhang, Z. \& Cao, J. Pollen wall development: the associated enzymes and metabolic pathways. Plant biology 15, 249-263, doi:10.1111/j.1438-8677.2012.00706.x (2013).

54. de Azevedo Souza, C. et al. A novel fatty Acyl-CoA Synthetase is required for pollen development and sporopollenin biosynthesis in Arabidopsis. The Plant cell 21, 507-525, doi:10.1105/tpc.108.062513 (2009).

55. Grienenberger, E. \& Legrand, M. Analysis of TETRAKETIDE $\alpha$-PYRONE REDUCTASE function in Arabidopsis thaliana reveals a previously unknown, but conserved, biochemical pathway in sporopollenin monomer biosynthesis. The Plant cell 22, 4067-4083 (2010).

56. Lallemand, B., Erhardt, M., Heitz, T. \& Legrand, M. Sporopollenin biosynthetic enzymes interact and constitute a metabolon localized to the endoplasmic reticulum of tapetum cells. Plant physiology 162, 616-625 (2013).

57. Wang, M., Li, Q., Di, F. S. \& Fischer, R. Expression of strictosidine synthase in selected subcellular compartments of tobacco plant. Acta Botanica Sinica 44, 579-582 (2002)

58. Schultz, J., Milpetz, F., Bork, P. \& Ponting, C. P. SMART, a simple modular architecture research tool: identification of signaling domains. Proceedings of the National Academy of Sciences of the United States of America 95, 5857-5864 (1998).

59. Miao, J. et al. Targeted mutagenesis in rice using CRISPR-Cas system. Cell Research 23, 1233-1236 (2013).

60. Li, S. et al. The OsmiR396c-OsGRF4-OsGIF1 regulatory module determines grain size and yield in rice. Plant biotechnology journal 14, 2134 (2016).

61. Chen, Y., Zou, T. \& McCormick, S. S-Adenosylmethionine Synthetase 3 Is Important for Pollen Tube Growth. Plant physiology 172, 244-253, doi:10.1104/pp.16.00774 (2016).

62. Aya, K. et al. Gibberellin modulates anther development in rice via the transcriptional regulation of GAMYB. The Plant cell 21, 1453-1472, doi:10.1105/tpc.108.062935 (2009).

63. Chen, X., Liu, J., Cheng, Y. \& Jia, D. HEN1 functions pleiotropically in Arabidopsis development and acts in C function in the flower. Development 129, 1085-1094 (2002).

64. He, X. et al. Influence of an ER-retention signal on the N-glycosylation of recombinant human alpha-L-iduronidase generated in seeds of Arabidopsis. Plant molecular biology 79, 157-169, doi:10.1007/s11103-012-9902-5 (2012).

\section{Acknowledgements}

This work was supported by the National Natural Science Foundation of China (no. 91435102, 31570004, and 31471474), and the Open Research Fund of the State Key Laboratory of Hybrid Rice (Hunan Hybrid Rice Research Center, 2016KF10), and Sichuan Provincial Funding for Distinguished Young Scholars (2015JQ0048).

\section{Author Contributions}

S.L. and P.L. designed and directed the experiments. D.C., Q.L. and Yanling L. performed the expression analysis, tissue localization and subcellular localization. J.Z., Q.D. and S.W. performed the genetic transformations. M.L., T.W. and Q.X. performed the phenotypic characterization of the mutant and the transgenic plants. Yueyang L., A.Z. and L.W. constructed all the vectors. T.Z. performed the cloning and functional analysis and collected almost all the data. T.Z. and S. L. analyzed the data, and wrote the main manuscript.

\section{Additional Information}

Supplementary information accompanies this paper at doi:10.1038/s41598-017-07064-4

Competing Interests: The authors declare that they have no competing interests.

Accession codes: The RGAP gene ID for OsSTRL2 is LOC_Os03g15710.

Publisher's note: Springer Nature remains neutral with regard to jurisdictional claims in published maps and institutional affiliations.

Open Access This article is licensed under a Creative Commons Attribution 4.0 International License, which permits use, sharing, adaptation, distribution and reproduction in any medium or format, as long as you give appropriate credit to the original author(s) and the source, provide a link to the Creative Commons license, and indicate if changes were made. The images or other third party material in this article are included in the article's Creative Commons license, unless indicated otherwise in a credit line to the material. If material is not included in the article's Creative Commons license and your intended use is not permitted by statutory regulation or exceeds the permitted use, you will need to obtain permission directly from the copyright holder. To view a copy of this license, visit http://creativecommons.org/licenses/by/4.0/.

(C) The Author(s) 2017 